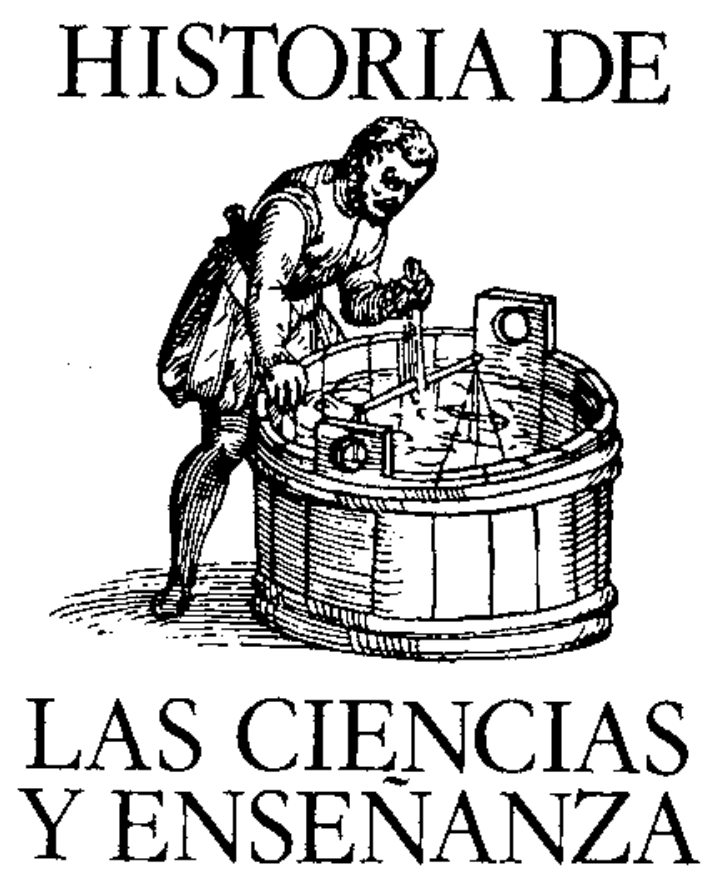

\title{
SEMEJANZAS Y DIFERENCIAS ENTRE LAS CONCEPCIONES INFANTILES Y LA EVOLUCION HISTORICA DE LAS CIENCIAS: EL EJEMPLO DEL CONCEPTO DE FUERZA Y ESPECIALMENTE DEL DE FUERZA DE GRAVEDAD
}

SANMARTI, N. y CASADELLA, J.

Departamento de Didáctica de las Ciencias y las Matemáticas. Universitat Autònoma de Barceiona. Bejlaterra.

\section{SUMMARY}

This research refers to the evolution of learning the concept of gravity. The previous ideas of the students, aged 12-13, were analysed to be taken into account prior to the methodology design.

An imaginary character defending the students' views was introduce to raise polemics.

Among other results, we want to outline that the theory of «impetus» eventually come out of the students and was coherently formulated.

\section{INTRODUCCION}

Numerosos estudios de didáctica (Gilbert y Watts 1983, Driver y Erikson 1983, Osborne y Al. 1983, Viennot 1979) ponen de manifiesto que ante los temas de estudio los alumnos exhiben lo que se ha llamdo pre. conceptos, razonamientos espontáneos o ideas propias; esto es, una nube de conceptos asumidos por elios, ge- neralmente erróneos, y de los que les resulta dificil desprenderse.

Asimismo es ampliamente reconocido que, si no se altera de manera consistente la estructura de los preconceptos en el proceso educativo, los conceptos más an- 
tiguos vuelven a ser utilizados en la interpretación de los fenómenos.

La mecánica se ha visto privilegiada como un tema sobre el cual se han investigado los preconceptos de los jóvenes estudiantes, probablemente por el amplio uso que se hace de su enseñanza (Nussbaum y Novak 1976. Watts y Zylberztajn 1981, Gunstone y White 1980 , 1981, Mc. Dermott 1984, Gunstone 1984). Por esta razón, es dificil que este trabajo sea enteramente original, tanto en el diseño como en las conclusiones.

La experiencia que describimos en este articulo (curso 1984-1985), se ha centrado en el aprendizaje del concepto de fuerza, relacionado con el cambio de movimiento y con la gravitación universal. Lo que se pretendia era utilizar el sistema solar como contexto fenomenológico, en el que los conceptos mencionados tienen un importante papel explicativo.

Es evidente que los estudiantes tienen grandes dificultades para asimilar estos contenidos. Pero queríamos superar la concepción de las fuerzas que se desprende del estudio de la estática, para introducir su papel en el movimiento de los cuerpos, tanto si son simples aproyectiles" como si son planetas.

Partiendo de la hipótesis de que las principales dificultades de los alumnos provienen de la superación de sus propios conceptos, físicos en este caso, consideramos como método apropiado hacerlos salir a la luz mediante el uso de la polémica. Para estimular esta polémica introdujimos un personaje imaginatio, llamado coloquialmente "Esotérix", que defendió, no hace falta decirlo, los errores conceptuales de los estudiantes que nos parecieron más destacables.

No teníamos como objetivo la completa asimilación de los conceptos introducidos en la clase. Creiamos que la evolución de las ideas, en los individuos como en la historia, se produce mucho más por una serie de transformaciones significativas que por dramáticas revelaciones repentinas, tal y como manifiesta I.B. Cohen (1980). En coherencia con esta presunción lo que pretendiamos, con esta experiencia didáctica, era hacer evolucionar los conceptos propios de los alumnos en la línea de su gradual sustitución por los de la mecánica newtoniana. Conviene, posiblemente, destacar que ésta no es la última etapa del crecimiento y transformación de las ideas de los físicos sobre el mundo. En realidad no creemos que exista ninguna etapa última y definitiva. Por lo tanto, tampoco se trataba de lograr sustituir los conceptos de los estudiantes, considerados absolutamente erróneos, por otros conceptos, cuya corrección resultará incontestable.

\section{DISEÑO DE LA EXPERIENCIA}

El nivel de los estudiantes a los que se dirigian las actividades programadas era el correspondiente al séptimo de la enseñanza general básica. Y una edad, por lo tanto, de 12 a 13 años aproximadamente. Conviene tener en cuenta esta anotación, ya que los resultados de la experiencia son aplicables a esta edad, mejor dicho, a este período escolar.

\subsection{Método de trabajo}

Uno de los autores era profesor de la escuela, precisamente del séptimo curso. De manera que la experiencia fue llevada a cabo por parte del profesor ordinario que tenían los mismos estudiantes, sin producirse ninguna alteración por lo que atañe a las relaciones entre el grupo de clase y su maestro. Las actividades se desarrollaron dentro del horario escolar. Correspondian, de hecho, a un tema del programa de ciencias del curso, visto de manera poco habitual.

Se grabaron en vídeo las entrevistas a una docena de estudiantes, antes y después de las actividades didácticas de la clase, así como los aspectos más relevantes de las actividades de clase. Además de esta muestra del curso de séptimo, también se realizaron entrevistas a 8 estudiantes de octavo, que habian trabajado el tema con otros planteamientos didácticos.

\subsection{Análisis de la situación de partida}

Estudiando las opiniones de los estudiantes de séptimo de enseñanza básica sobre la gravitación universal y las fuerzas antes de trabajar el tema en clase llegamos a la conclusión de que nos permitian hacer una clasificación orientativa. Con el peligro de esquematizar en exceso, consideramos tres grupos:

a) Un reducido grupo de estudiantes demostraban tener, ante los fenómenos que les presentamos, un modelo en proceso de estructuración lógica. Sus respuestas eran encadenadas, hasta el punto de que ellos descubrían sus propias contradicciones al verbalizarlas.

Dentro de este grupo nos podriamos sorprender de algunas respuestas ingeniosas. Por ejemplo, una de las preguntas típicas que les haciamos era si el peso de una persona disminuiría al ir a las antípodas, haciéndolo en términos de "arriba o abajo de la tierra». Un estudiante matizó que en una tierra esférica no tenía sentido hablar de arriba o de abajo, y que incluso podría considerarse que, desde cualquier punto de la superficie de una esfera, abajo seria el centro.

También dentro de este grupo un estudiante, mientras vacilaba sobre las causas de la gravedad, indicaba que debía ser en función del volumen de los cuerpos, anladiendo poco después que además del volumen intervendria también su densidad. No deja de intrigar que Newton, para definir la masa, dijera que ésta surgía de la densidad y del volumen conjuntamente (PRINCIPIO 1687, definición primera).

Resulta realmente interesante la existencia de este grupito, entre otras cosas porque demuestra que no siempre hace falta considerar los conceptos y razonamientos propios de los alumnos como equivocados.

ENSEN̄ANZA DE LAS CIENCIAS, 1987, 5 (1) 
b) En un segundo grupo de estudiantes también se observó la existencia de modelos, pero su principal caracteristica consistia en su encuadramiento en lo que se ha llamado errores conceptuales. Algunos autores consideran que este tipo de modelos se encuentran fuertemente ligados a las intuiciones sugeridas por las experiencias sensoriales, sin que hayan pasado por la criba de la crítica o la reflexión.

Muchos de estos conceptos son extraordinariamente generales en la población estudiantil de todo e! mundo. Tal vez esto justifique que explicitemos algunos. Presentan una formulación parecida a la siguiente:

* Creer que el peso y la fuerza de atracción gravitatoria son cosas distintas.

* La caida de los graves no es debida a la acción de ningun tipo de fuerza.

* La fuerza es la causa del movimiento. Una fuerza constante provoca una velocidad uniforme.

- La no distinción entre fuerza, impulso, energía.

* El hecho de que los cuerpos no se detengan al momento de desaparecer la fuerza, es debido a que de alguna manera retienen la fuerza, o el impulso, o la energia, y la van perdiendo mientras corren.

* La rotación de la tierra tiene alguna relación con la catusa de la atracción gravitatoria.

- Los satélites y los planetas sólo pueden dar vueltas estables en determinadas regiones del espacio, conocidas como órbitas. Pueden ir más rápido o más lentamente por su órbita.

No todos los estudiantes de este grupo participan de las mismas concepciones. Incluso las opiniones de ca. da uno se pueden ver sometidas a modificaciones ocasionales. Por último la situación de un individuo en un grupo no es fija.

c) En un tercer grupo de estudiantes era muy difícil lograr que expresasen sus opiniones. Algunos de ellos manifestaban que nunca se habian planteado cuestiones parecidas, o que no eran capaces de imaginarse ninguna respuesta a los enigmas que se les presentaban. No descartamos que algunos de estos estudiantes tuvieran sus dificultades más importantes en la expresión verbal de sus ideas o intuiciones.

Esta falta de homogeneidad supone, en consecuencia, una dificultad suplementaria. Todos los planteamientos previos a la actividad didáctica apuntaban a la actuacion sobre un colectivo imaginario, compuesto únicamente por estudiantes del tipo encontrado en el segundo grupo. El material y sobre todo la atención dada a los alumnos se intentó que tuviera en consideración los tres grupos, de tal manera que cada uno de ellos tuviera la posibilidad de hacer algun progreso a partir de sus propios planteamientos.

\subsection{Programación de trabajo}

La introducción de los temas se llevaba a cabo de la ENSEÑANZA DE LAS CIENCIAS, 1987, 5 (1) mano del personaje "Esotérix», que desde el primer momento adoptaba un tono polémico, defendiendo sus opiniones e invitando a los asistentes a la discusión con él. Con la atención de articular la crítica o la defensa de las teorías del personaje "Esotérix», la clase se dividia en pequeños grupos de trabajo, que después comunicaban al gran grupo los resultados de sus discusiones, experiencias, etc. En el gran grupo algunos estudiantes quedaban al margen y no mostraban dema. siado interés, ya que una minoria conseguía dominar el debate. Pero, en cambio, en los pequeños grupos la participación era total, las discusiones más ricas, aunque también más desordenadas.

Se programaron actividades basadas en las siguientes cuestiones:

* ¿La tierra es redonda? ¿Cómo se aguanta el espacio? ¿Cómo se mueve?

* Las fuerzas de contacto y las de transmisión a distancia (con manipulación de imanes y limaduras de hierro). Composición y descomposición de las fuerzas. Efectos de las fuerzas y su medida.

* Movimiento uniforme y ley de inercia.

* Movimiento de caida libre. Las fuerzas y el movimiento.

- El movimiento de los proyectiles, de los satélites ar. tificiales, de la luna y los planetas.

* La presión: principio de Pascal. La presión atmos. férica y el peso.

El hecho de que las opiniones a debatir fueran las de Esotérix y no las del maestro rompia la dinámica de tomar las opiniones del profesor de una manera acrítica, estimulando la búsqueda de criterios propios para la credibilidad de determinadas explicaciones.

\section{ALGUNAS REFLEXIONES EN TORNO A LAS SEMEJANZAS Y DIFERENCIAS EN. TRE LAS CONCEPCIONES INFANTILES $Y$ LA EVOLUCION HISTORICA DE LAS CIENCIAS}

Después del análisis del comportamiento de los estudiantes, que se ha limitado al aspecto cualitativo, creemos haber observado las siguientes situaciones:

a) Los preconceptos de un estudiante, a esta edad, no forman en general un cuerpo coherente, defendido continuamente por él, o asimilado globalmente al estilo de los paradigmas de Kuhn. Lo prueba el hecho de que, en indeterminadas condiciones, cualquier estudiante ante un problema es capaz de ensayar diversas hipótesis "ad hoc». De hecho los jóvenes estudiantes son parti. cularmente dados a la invención de hipótesis, lo que valoramos positivamente, y nos podemos encontrar 0 con que las defiendan con uñas y dientes, o con que las cambien a cada momoento.

b) No obstante lo que se ha dicho anteriormente, hay 
algunos preconceptos radicalmente universales y fuertemente prefijados, como por ejemplo los que afirman que:

* La aceleración de caida libre está relacionada con la masa del cuerpoo que cae.

* El movimiento precisa de fuerzas para mantenerse.

* El impulso inicial se queda dentro del cuerpo en movimiento, consumiéndose a la vez que éste avanza, perdiendo, en consecuencia, velocidad continuamente has" ta pararse.

c) Las experiencias sensoriales pueden actuar como un obstáculo de algunos conceptos. Así, por ejemplo, la adaptación del organismo al campo gravitatorio hace que no notemos que el peso es una fuerza que nos aprieta sistemática y permanentemente contra la superficie de la tierra. Igualmente la presión atmosférica nos comprime sin que tengamos ningún tipo de sensación. En cambio las fuerzas que puedan aplicarse las personas entre si, o los impactos, etc. dan una respuesta sensorial clara.

Había estudiantes que no consideraban el peso como una fuerza, al menos una fuerza de la misma categoría de las que intervienen al estirar una cuerda, o dar un empujón.

Por lo que se refiere a la atmósfera, los niftos y niñas se veían como rodeados por ella. De esta manera veían como algo muy natural el que ésta impidiera la caída al espacio exterior. No se trataba, entonces, de que hiciera una fuerza efectiva o una presión sobre nosotros y las cosas. Nos inclinamos a creer que la atmósfera parecía actuar como un obstáculo que impedía que fuéramos alejados de la tierra. De todas maneras, los preconceptos relativos al papel de la atmósfera como causa de la gravedad no pueden ser interpretados tomando como marco de referencia los conceptos científicos profesados por el maestro, que se tienen que conside. rar más evolucionados.

Los ejemplos anteriores son de un tipo en el que la falta de percepción sensorial dificulta la formación de un concepto. Pero puede haber percepciones positivas que inducen directamente conceptos equivocados. Dentro de esta clase se podria incluir las representaciones mentales de las informaciones pasadas por los "mass media». Así por ejemplo colocar un satélite artificial en órbita, dejar el artefacto y conectar el motor para que gire alrededor de la tierra. De manera parecida, las experiencias de los astronautas sobre la ingravedad, cuando su nave orbita la tierra, pueden inducir a pensar que alli donde se encuentran no hay gravedad. Una frontera "natural» para la fuerza gravitatoria podría ser la misma que la de la atmósfera.

d) Cuando un estudiante defiende una determinada hipótesis, en la que cree sinceramente, puede ser capaz de prolongarla en conclusiones lógicas. Pero de aqui no se puede deducir que este estudiante defiende una teoría eleborada completamente. Así, por ejemplo, creemos que no tiene demasiado sentido decir que los estudiantes son aristotélicos o cualquier otra cosa, por el hecho de que en algunas ocasiones sus conceptos puedan identificarse con los de la Filosofía aristotélica. Más que la pertenencia a un cuerpo teórico elaborado, los conceptos, en estas edades, parecen caracterizarse por la falta de estructura y por la dispersión. Esto provoca que no aparezcan ni desaparezcan en bloque.

Hay autores, como por ejemplo Saltiel, E. y Viennot, L. (1985) que recomiendan la búsqueda de una estruc. tura interna en el seno de las concepciones generadas por razonamiento espontáneo. En el trabajo que citamos, consideran que existen conexiones entre las «teorias" de los estudiantes y las teorías preclásicas correspondientes al mismo tema, como si de alguna manera se volviera a revivir individualmente las principales eta. pas del desarrollo histórico. Aunque sin abandonar esta hipótesis, se formulan ciertas reservas sobre la conveniencia de interpretar demasiado estrechamente el paralelismo entre preconceptos y las teorías de un determinado período histórico. Las reservas las compartimos plenamente.

Nuestros alumnos demostraban tener conceptos sobre el movimiento y la fuerza (impulso o energía) fácilmente comparables con las teorias escolásticas del «impetus». Pero al lado de éstos defendían otros, como por ejemplo la rotación de la tierra, y la atmósfera, como causa de la atracción gravitatoria, que son dificilmente identificables con alguna teoria histórica.

Es muy probable que en la génesis de cualquier hipótesis, tanto a nivel de nuestros estudiantes como de cualquier pensador a lo largo de la historia, intervengan una serie de factores poco variables en el transcurso de los últimos milenios. Esta hipótesis podría explicar, en principio, que personas separadas por ambientes culturales tan distantes, como por ejemplo los estudiantes actuales y los filósofos de Grecia o de la edad media, puedan formular conceptos parecidos cuando se enfrentan a problemas «similares».

No obstante, pensamos que la historia de las ciencias resulta un instrumento valioso para los profesores: de entrada ofrece nuevos horizontes conceptuales, am. pliando los utilizados para la formación inicial en los casos en que la historia de las ciencias no forma parte del plan de estudios; permite en particular ver cómo conceptos diferentes de los actuales se han articulado de manera coherente, han sido defendidos por personajes ilustres y han resultado difíciles de superar. Todo esto ayuda a no menospreciar las concepciones espontáneas de los alumnos.

Por otro lado la historia de las ciencias permite ver cómo ésta se construye en un proceso vivo, en el que el razonamiento lógico no es el único que juega un papel destacado. También las intuiciones, la formación anterior, las concepciones metafísicas, los resultados de experimentos debidamente planificados al lado de conocimientos empíricos ancestrales interpretados de una 
experimentos debidamente planificados al lado de conocimientos empíricos ancestrales interpretados de una manera totalmente nueva, son elementos decisivos en este proceso constructivo-destructivo. De alguna manera tiene que haber una relación entre el crecimiento dialéctico de la ciencia de la colectividad y la reconstrucción del conocimiento individualizado en nuest ros alumnos. Incluso las incoherencias de éstos tienen su paralelo histórico.

Por último, los conocimientos que ofrece la historia, permiten hacer precisiones de carácter epistemológico. Por ejemplo, al no haber ninguna teoria que pueda durar siempre (Holton y Brush), las concepciones "cientificas» de! profesor no deberán tener mejor suerte, en último término, que las de sus alumnos. Resulta que es un poco absurdo calificar los conceptos de los alumnos como equivocados, por oposición a otros que tampoco son definitivamente ciertos. Lo cual no significa que en la historia de las ideas no se establezca una se. lección natural, comparable a la de las especies, que nos permite una cierta «jerarquia» entre las diversas teorias científicas.

e) En general los alumnos se incorporaron ampliamente a las discusiones, especiaimente en las de grupo pequeño. Un reducido número de alumnos, que coincidia con los que tendían a memorizar sus aprendizajes, quedaron un poco al margen del debate. El resto, donde habia niños de los tres grupos iniciaimente detectados, se lanzaron virtualmente a elucubrar, a pesar de que a menudo lo hacian de forma desordenada.

En el test final y en las entrevistas que se realizaron a chicos y chicas, se pudo constatar que se habian conseguido los conocimientos minimos en cuanto a la medida de las fuerzas, composición y otros tópicos habituales del programa de séptimo. Además eran capaces de relacionar fenómenos diversos, aparentemente diferenciados, aunque fuera a nivel intuitivo, que habian sido trabajados en clase con el fin de introducir estos tópicos.

f) Es muy difícil que los niños abandonen sus conceptos para adoptar los aceptados por la comunidad científica. Algunas de las dificultades para este cambio conceptual podrían ser las siguientes:

* La dificultad de distinguir entre explicaciones diversas, incluso contradictorias, sobre un mismo fenómeno. Muchos alumnos manifestaban un acuerdo solidario con los compañeros excesivamente rápido, sin percatarse del carácter divergente de sus afirmaciones y las propias. Por este mecanismo se dificulta la crítica de las ideas que se mantienen al cubierto de las opuestas.

* Al contrario de las teorías del falsacionismo de Popper, según las cuales las teorías no son verdaderas y las vigentes mantienen su valor gracias a que no ha sido demostrada su falsedad, la mayor parte de los niños de esta edad creen en la verosimilitud de sus hipótesis, aunque sean contradictorias con otras que también profesen o con hechos manifiestos que las contradicen. Es necesaria la educación del método para conseguir que los estudiantes sean conscientes de la necesidad de descartar concepciones.

g) No deja de ser intrigante que la teoria del «impetus» apareciera al final de las actividades didácticas inalterada; o mejor dicho limpia de muchas otras for* mulaciones que fueron asumidas y después abandonadas por muchos estudiantes. En parte podría ser debido a que nosotros no supimos distinguir esta «preteoria» en las entrevistas iniciales, y en consecuencia no incluimos actividades sistemáticas para hacerla evolucionar. Pero falta saber si efectivamente resulta ser una de estas concepciones extrañamente persistentes, tanto en la historia de la cultura como en la evolución conceptual de nuestros alumnos, tal como sugieren Saltiel y Viennot (1985).

\section{REFERENCIAS BIBLIOGRAFICAS}

COHEN, I.B., 1980, La revolución newtoniana y la transformación de las ideas cientificas. (Alianza Ed: Madrid).

DRIVER, R., ERICKSON, G., 1983, Theories-in-action: Some theoretical and empirical issues in the study of students' conceptuals frameworks in science. Stud. Sci. Educ., 10, 37-60.
GILBERT, J., WATTS, M., 1983, Concepts, misconceptions and afternative conceptions: Changing perspectives in science education. Stud. Sci. Educ., 10,, 61-98.

GILBERT, J.K., ZYLBERSZTAJN, A.. 1985, A conceptual framework for science education: The case study of for* ce and movement. Eur. J. Sci. Educ., 7 (2), 107-120. 
GUNSTONE, R., 1984, Circular motion: Some pre-instroution alternative frameworks. Res. in Sci. Educ. 14, 125-136.

GUNSTONE, R., WHITE, R., 1981, Undestanding of gravity. Sci. Educ., 65, (3), 291-299.

GUNSTONE, R., WHITE, R., 1980, A matter of gravity. Res, in Sci. Educ., 10, 35-44.

HOLTON, BRUSH, 1976, Introducción a los conceptos y teorías de las ciencias físicas, (Reverté: Barcelona).

MALi, G., HOWE, A., 1979, Development of Earth and gra vity concepts among Nepali children. Sci. Educ., 63 (5), 685.691 .

MCDERMOTT, L.C., 1984, Research in conceptual understanding of mechanics. Phys. Today, 37, 23-32.
NUSSBAUM, J., NOVAK, J., 1976, An assessment of children's concepts of the earth utilizing structured interviews. Sci. Educ., 60 (4), 535-550.

SALTIEL, E., VIENNOT, L., 1985, ¿Qué aprendemos de las semejanzas entre jas ideas históricas y el razonamiento espontáneo de los estudiantes?. Enseñanza de las Ciencias, $3(2), 137-144$.

VIENNOT, L., 1979, Spontaneous reasoning in elementary dynamics. Eur. J. Sci. Educ., I (2), 205-221.

WATTS, D.M., 1983, A study of schoolchildren's alternative frameworks of the concepte of force. Eur. J. Sci. Educ., 5 (2), 217.230.

WATTS, D.M., ZYLBERSZTASN, A., 1981, A survey of some children's ideas about force. Phis. Educ., 16 (6), 360-365. 